\title{
Transient receptor potential channel TRPV4 mediates TGF- $\beta 1$-induced differentiation of human ventricular fibroblasts
}

\author{
Min-Soo Ahn ${ }^{1}$, Young Woo Eom ${ }^{2}$, Ji-Eun $\mathrm{Oh}^{2}$, Seung-Kuy Cha ${ }^{3}$, Kyu Sang Park ${ }^{3}$, \\ Jung-Woo Son ${ }^{1}$, Jun-Won Lee ${ }^{1}$, Young Jin Youn ${ }^{1}$, Sung Gyun Ahn ${ }^{1}$, \\ Jang-Young $\mathrm{Kim}^{1}$, Seung-Hwan Lee ${ }^{1}$, Junghan Yoon ${ }^{1}$, Byung-Su Yoo ${ }^{1}$ \\ ${ }^{1}$ Cardiology Division, Department of Internal Medicine, \\ Yonsei University Wonju College of Medicine, Wonju, Korea \\ ${ }^{2}$ Cell Therapy and Tissue Engineering Center, Yonsei University Wonju College of Medicine, Wonju, Korea \\ ${ }^{3}$ Department of Physiology, Yonsei University Wonju College of Medicine, Wonju, Korea
}

\begin{abstract}
Background: Cardiac fibroblasts (CFs) are principal extracellular matrix-producing cells. In response to injury, CFs transdifferentiate into myofibroblasts. Intracellular calcium $\left(\mathrm{Ca}^{2+}\right)$ signaling, involved in fibroblast proliferation and differentiation, is activated in fibroblasts through transient receptor potential $(T R P)$ channels, but the function of these channels has not been investigated in human ventricular CFs. Under evaluation in this study, was the role of TRP channels in the differentiation of human ventricular $C F$ induced by transforming the growth factor beta (TGF- $\beta$ ), a pro-fibrotic cytokine.

Methods: Human ventricular CFs were used in this study. The differentiation of CFs into myofibroblast was induced with TGF- $\beta$ and was identified by the expression of smooth muscle actin.

Results: Results indicate that $\mathrm{Ca}^{2+}$ signaling was an essential component of ventricular $C F$ differentiation. CFs treated with TGF- $\beta$ demonstrated increased expression of a TRP channel, TRPV4, both at the mRNA and protein levels, which corresponded with CF-myofibroblast trans-differentiation, as evidenced by the upregulation of $\alpha$-smooth muscle actin, a myofibroblast marker, and plasminogen activator inhibitor-1, which are fibrogenesis markers. An agonist of TRPV4 induced the conversion of $C F s$ into myofibroblasts, whereas it's antagonist as well a $\mathrm{Ca}^{2+}$ chelating agent reduced it, indicating that the $\mathrm{Ca}^{2+}$ influx throughTRPV4 is required for CF trans-differentiation. Overall, these results demonstrate that TRPV4-mediated $\mathrm{Ca}^{2+}$ influx participates in regulating the differentiation of human ventricular CFs into myofibroblasts through the MAPK/ERK pathway.

Conclusions: Overall, these results demonstrate that TRPV4-mediated $\mathrm{Ca}^{2+}$ influx participates in regulating the differentiation of human ventricular CFs into myofibroblasts through the MAPK/ERK pathway. (Cardiol J 2020; 27, 2: 162-170)
\end{abstract}

Key words: fibroblast, myofibroblast, transient receptor potential channels, calcium

\section{Introduction}

Cardiac fibroblasts (CFs) residing in the interstitial space comprise the largest cell population in the myocardium and provide structural support for cardiomyocytes by producing an extracellular matrix (ECM) which maintains heart architecture under physiological conditions [1]. CFs also play a key role in response to heart injury. Upon pathological stimuli, CFs differentiate into myofibroblasts

Address for correspondence: Byung-Su Yoo, MD, PhD, Cardiology Division, Department of Internal Medicine, Wonju College of Medicine, Yonsei University, 20 Ilsan-ro, Wonju, Korea, tel: 82-33-741-0917, fax: 82-33-741-1219, e-mail: yubs@yonsei.ac.kr

Received: 26.12.2018Ａccepted: 4.05.2019 
characterized by contractility and overproduction of ECM, which causes wound retraction and healing by replacing necrotized cardiomyocytes. However, excessive activity of myofibroblasts can cause scarring and cardiac fibrosis followed by heart hypertrophy, enlargement of cardiac chambers, cell apoptosis, and ultimately heart failure [2].

Myofibroblasts are absent in normal cardiac tissue and only appear following injury [3]. The differentiation of CFs into myofibroblasts is a crucial process in heart remodeling, and it is established that the transforming growth factor (TGF)- $\beta 1$ secreted by inflammatory cells directly induces a switch from the quiescent phenotype of CFs to active synthetic and contractile phenotype of myofibroblasts $[4,5]$. The latter can be distinguished from $\mathrm{CFs}$ by the expression of several smooth muscle cell markers, including smooth muscle actin ( $\alpha$-SMA), which provide myofibroblast contractility $[6,7]$.

Although a number of signaling pathways are known to mediate fibroblast-to-myofibroblast conversion and fibrogenesis in the heart, there are few effective therapies to treat cardiac fibrosis, emphasizing the need to identify new molecular targets. $\mathrm{Ca}^{2+}$ signals regulate diverse cellular functions, and are known to be involved in fibroblast proliferation and ECM synthesis [8]. Fibroblasts have a relatively depolarized resting membrane potential, which is between -31 and $-16 \mathrm{mV}$ in atrial fibroblasts [9], and lack functional voltagegated $\mathrm{Ca}^{2+}$ channels [10]. Instead, they express transient receptor potential (TRP) channels which provide nonselective intracellular $\mathrm{Ca}^{2+}$ flux and are widely present in many cell types [11]. Recently four TRP channels; TRPM7, TRPC3, TRPC6, and TRPV4, were found to be essential for myofibroblast trans differentiation [12-15]. However, their function appears to be species- and tissue-specific and it is not known whether TRP-mediated $\mathrm{Ca}^{2+}$ signaling is specifically required for the conversion of human ventricular CFs into myofibroblasts [16]. Therefore, the aim of this study was to investigate the role of TRP channels in the TGF- $\beta$-mediated transformation of human ventricular CFs.

\section{Methods}

\section{Materials}

Normal human ventricular cardiac fibroblasts (NHCF-V) (Product code; CC-2904) were purchased from Lonza (Walkersville Inc., MD, USA). An intracellular calcium chelator BAPTA$\mathrm{AM}$, a selective antagonist ( $\mathrm{RN}-9893)$, agonist
(GSK1016790A) of TRPV4 and selective inhibitor of MAPK/ERK kinase (U0126) were purchased from Sigma-Aldrich (St. Louis, MO, USA) [17]. Polyclonal antibodies against TRPV4 were obtained from Alomone Labs (Jerusalem, Israel), plasminogen activator inhibitor-1 (PAI-1)-specific antibody was from Santa Cruz Biotechnology (Santa Cruz, CA, USA), $\alpha$-SMA antibody was from Abcam (Cambridge, MA, USA), and monoclonal antibodies against phosphorylated (p)-ERK and total ERK were obtained from Cell Signaling Technology (Danvers, MA, USA). TGF- $\beta$ was purchased from PeproTech (Rocky Hill, NJ, USA).

\section{Cell culture and TGF- $\beta$ treatment}

Cardiac fibroblasts were cultured in fibroblast growth medium (FGM, Lonza, Basel, Switzerland) supplemented with fetal calf serum (FCS), streptomycin $(50 \mu \mathrm{g} / \mathrm{mL})$, and penicillin $(100 \mathrm{U} / \mathrm{mL})$ at $37^{\circ} \mathrm{C}$ in a humidified atmosphere of $5 \% \mathrm{CO}_{2}$. After $24 \mathrm{~h}$ starvation in serum-free FGM, the medium was replaced by FGM containing $2 \mathrm{ng} / \mathrm{mL}$ recombinant human TGF- $\beta$. CFs were cultured for different times $(0,6,12,24$, and $48 \mathrm{~h})$.

\section{Polymerase chain reaction}

Gene-specific primers were used to detect the expression of human TRP channels as described previously [14]. Ventricular CFs were seeded into $10-\mathrm{cm}$ dishes, cultured in serum-free FGM for $24 \mathrm{~h}$, and then stimulated with TGF- $\beta(2 \mathrm{ng} / \mathrm{mL})$ for $3,6,12,24$, and $48 \mathrm{~h}$. Cells were collected, and total RNA was extracted by Trizol (Invitrogen, Paisley, UK), and cDNA was synthesized, which was used as a template for the polymerase chain reaction (PCR). The amplified products were resolved by electrophoresis on $2 \%$ agarose gels and photographed using the FluorChem FC2 system (Cell Biosciences, Santa Clara, CA, USA).

\section{Western blotting}

Cardiac fibroblast differentiation was induced with $2 \mathrm{ng} / \mathrm{mL}$ TGF- $\beta$ in the absence or presence of $1 \mu \mathrm{M} / \mathrm{mL} \mathrm{Ca}^{2+}$ chelator BAPTA-AM for $48 \mathrm{~h}$, or different concentrations of TRPV4 agonist GSK1016790A (10, 20 and $50 \mathrm{nM})$ or antagonist RN-9893 (10, 20, and $50 \mu \mathrm{M})$ for $24 \mathrm{~h}$. DMSO was used as the vehicle control. For inhibition of ERK signaling, CFs were treated with U0126 $(10 \mu \mathrm{M})$, an inhibitor of MAPK/ERK kinase for $48 \mathrm{~h}$. CFs were homogenized and lysed in RIPA buffer containing $50 \mathrm{mM}$ Tris $\mathrm{pH} 7.4,150 \mathrm{mM} \mathrm{NaCl}, 1 \%$ NP40, $0.5 \%$ sodium deoxycholate, $0.1 \%$ SDS, and protease inhibitor cocktail (Sigma, St. Louis, MO, 
USA). Protein concentration in cell lysates was determined by the Bradford assay (Bio-Rad, Richmond, CA, USA). Proteins were separated in 10\% SDS-PAGE gels under reducing conditions, electrophoretically transferred onto equilibrated polyvinylidene difluoride membranes (Immobilon-P, Millipore, Billerica, MA, USA), and probed with primary antibodies against TRPV4, $\alpha$-SMA, p-ERK (T202/Y204), total ERK, PAI-1, and $\beta$-actin was used as an internal control. Reactivity to the anti-PAI-1 antibody was also determined in the cell culture supernatant.

\section{Measurement of cytosolic $\mathrm{Ca}^{2+}$}

$\mathrm{Ca}^{2+}$ concentration was measured using a ratiometric indicator Fura2 as described previously [18]. CFs were seeded onto black-walled 96 -well plates $\left(5 \times 10^{4}\right.$ cells/well $)$ and treated with $2 \mathrm{mM}$ $\mathrm{Ca}^{2+}$ with or without TGF- $\beta$ for $24 \mathrm{~h}$ to induce differentiation into myofibroblasts. Cells were loaded with $5 \mu \mathrm{M}$ Fura2/AM (Invitrogen, Carlsbad, CA, USA) in Krebs-Ringer bicarbonate buffer $(126 \mathrm{mM}$ $\mathrm{NaCl}, 4 \mathrm{mM} \mathrm{KCl}, 10 \mathrm{mM}$ HEPES, $1 \mathrm{mM} \mathrm{MgCl}$, $5.5 \mathrm{mM}$ glucose, $\mathrm{pH} 7.4$ ) for $30 \mathrm{~min}$ at $37^{\circ} \mathrm{C}$, and fluorescence was measured at $510 \mathrm{~nm}$ in response to alternate excitation at $340 \mathrm{~nm}$ and $380 \mathrm{~nm}$ using a Flexstation fluorescence reader (Molecular Devices, Sunnyvale, CA, USA). The changes in the Fura2 fluorescence ratio $\left(\mathrm{F}_{340} / \mathrm{F}_{380}\right)$ caused by $2 \mathrm{mM} \mathrm{Ca}^{2+}$ were estimated by the area under the curve (AUC) and normalized to that caused by $10 \mu \mathrm{M}$ ionomycin-elicited $\mathrm{Ca}^{2+}$ signal. To assess the effects of TRPV4 activation, CFs were treated with $100 \mathrm{nM}$ GSK1016790A.

\section{Statistical analysis}

Statistical analysis was performed with oneway ANOVA followed by the Tukey post hoc analysis or Student t-test; changes at $\mathrm{p}<0.05$ were considered statistically significant. All analyses were performed using SPSS v18 (SPSS, Chicago, IL, USA).

\section{Results}

TGF- $\beta$ induces $\mathrm{Ca}^{2+}$-dependent differentiation of ventricular fibroblasts and expression of $\mathrm{Ca}^{2+}$-permeable channel TRPV4

To determine whether $\mathrm{Ca}^{2+}$ was required for TGF- $\beta$-mediated CF differentiation into myofibroblasts, TGF- $\beta$-stimulated cells were treated with the intracellular $\mathrm{Ca}^{2+}$ chelator BAPTA-AM and the expression of myofibroblast differentiation marker $\alpha$-SMA (Fig. 1) was analyzed. The expression of $\alpha$-SMA was suppressed with intracellular $\mathrm{Ca}^{2+}$ chelator. The results showed that chelation of intracellular $\mathrm{Ca}^{2+}$ inhibited $\mathrm{CF}$ differentiation, indicating that $\mathrm{Ca}^{2+}$ signaling was essential for $\mathrm{CF}$ conversion into myofibroblasts.

To determine which $\mathrm{Ca}^{2+}$ channel is responsible for the TGF- $\beta$-induced differentiation of human ventricular CFs, the expression of TRP channels in CFs were examined. TRPV4 was the only TRP channel upregulated by TGF- $\beta 1$ both at the mRNA (Fig. 2) and protein (Fig. 3) levels, which corresponded to the induction of $\mathrm{CF}$ differentiation into myofibroblasts manifested by an increased expression of $\alpha$-SMA and PAI-1 (Fig. 3). PAI- 1 is the main inhibitor of serine proteases urinary and tissue plasminogen activators, which are responsible for plasmin formation, and of plasmin-dependent matrix metalloproteinases. Since plasmin and matrix metalloproteases degrade the ECM, their downregulation promotes ECM deposition; therefore, PAI-1 is considered a biomarker of excessive fibrogenesis and is implicated in the pathophysiology of fibrosis [19].

\section{TRPV4 is required for the differentiation of ventricular fibroblasts}

To determine the involvement of TRPV4 in myofibroblast trans-differentiation, CFs were cultured with TRPV4 agonist GSK1016790A or antagonist RN-9893 and were then stimulated with TGF- $\beta$ for $24 \mathrm{~h}$. The results indicate that the TRPV4 agonist significantly promoted and TRPV4 antagonist suppressed the conversion of CFs into myofibroblasts, as evidenced by dose-dependent increase and decrease, respectively, in $\alpha$-SMA expression (Fig. 4).

\section{TRPV4 downstream signaling is mediated by ERK}

The data obtained indicates that TRPV4 is involved in TGF- $\beta$-stimulated CF differentiation. Therefore, potential downstream signaling mechanisms mediating TRPV4 activity were subsequently addressed. Since it is known that fibroblast differentiation is induced by reactive oxygen species (ROS) and inhibited through mitogen-activated protein kinase (MAPK)/ERK signaling, and thus ERK1/2 phosphorylation in TGF- $\beta$-induced CFs treated with $1 \mu \mathrm{M}$ of $\mathrm{Ca}^{2+}$ chelator BAPTA-AM was analyzed. ERK phosphorylation induced by TGF- $\beta$ during the first hour of treatment was suppressed in a time-dependent manner by BAPTA-AM, which did not affect ERK activation at the early phase, but 


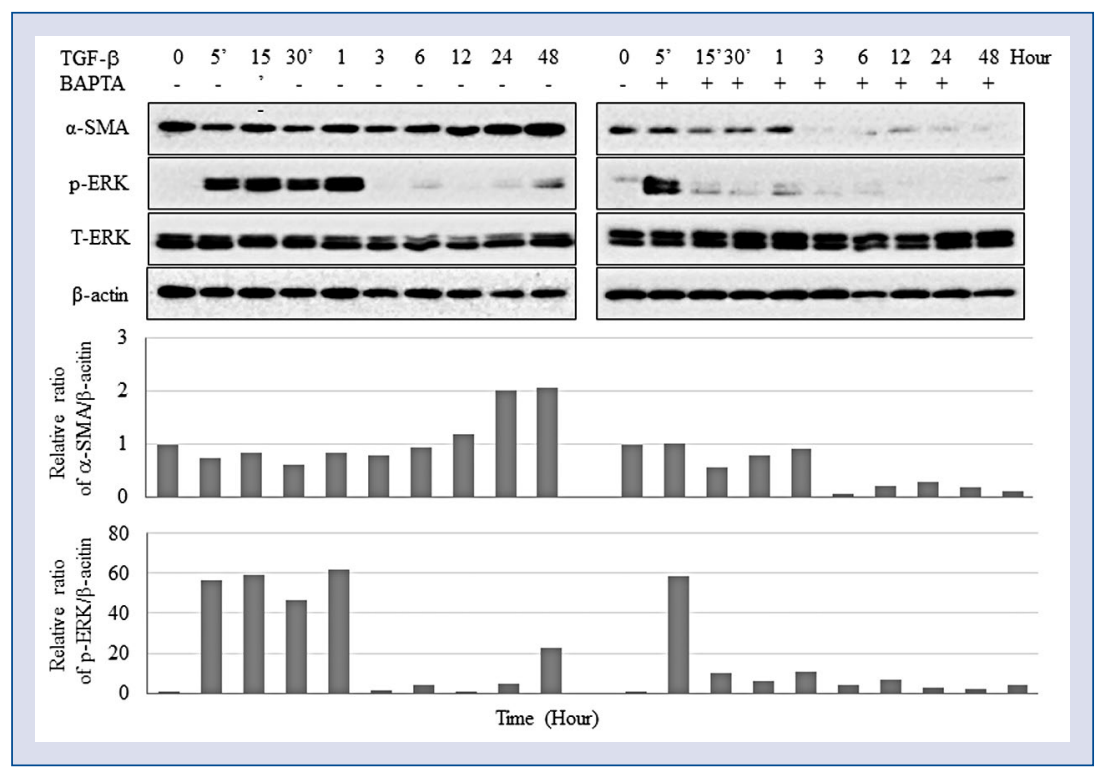

Figure 1. Fibroblast differentiation depends on $\mathrm{Ca}^{2+}$. Cardiac fibroblasts induced with $2 \mathrm{ng} / \mathrm{mL}$ transforming growth factor beta (TGF- $\beta$ ) were treated or not with $1 \mu \mathrm{g} / \mathrm{mL}$ BAPTA-AM and analyzed for protein expression by Western blotting.

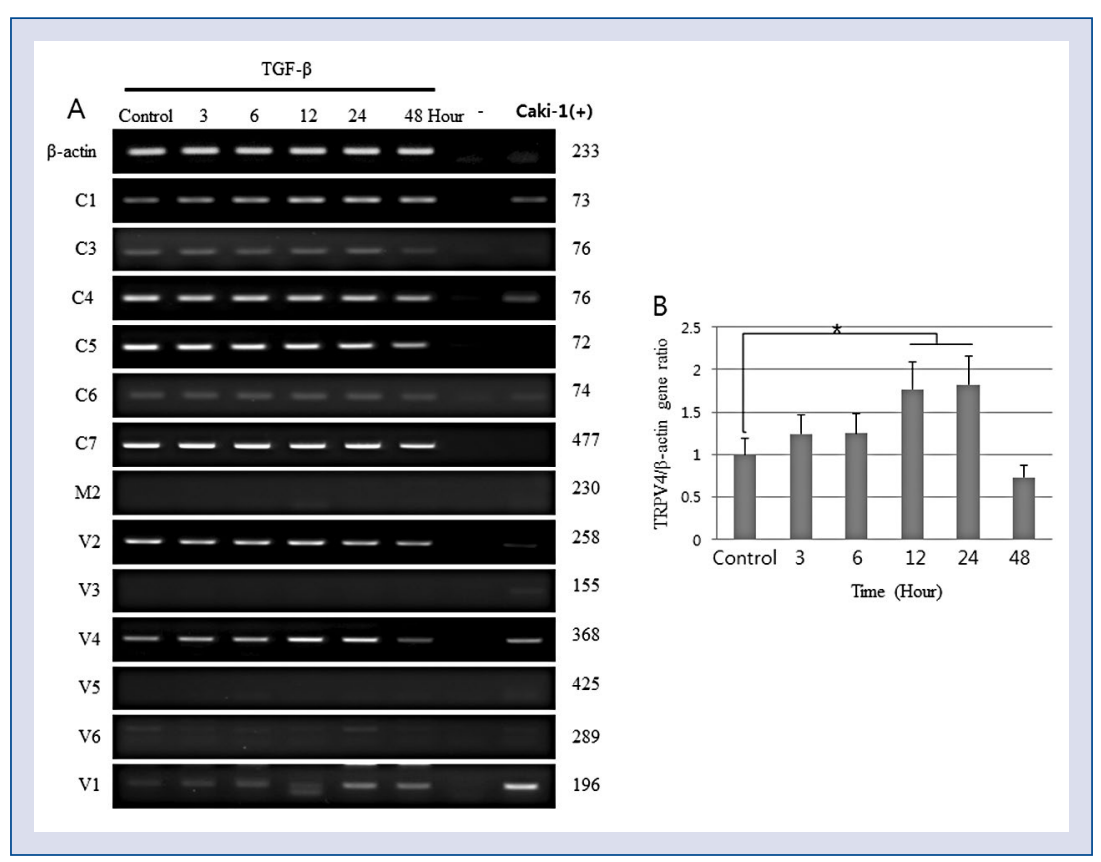

Figure 2. Transforming growth factor beta (TGF- $\beta$ ) induces TRPV4 mRNA expression. Cardiac fibroblasts were treated with $2 \mathrm{ng} / \mathrm{mL}$ TGF- $\beta$ and analyzed for the expression of transient receptor potential (TRP) channels by a polymerase chain reaction (PCR); A. Representative image of an agarose gel showing PCR amplification products; B. Semi-quantitative analysis of TRPV4 mRNA expression relative to $\alpha$-actin ( ${ }^{*} p<0.05$ vs. untreated cells).

inhibited it at the late phase (Fig. 1). Furthermore, ERK inhibitor U0126 caused the same effects as shown by TRPV4 antagonist RN-9893, since both these suppressed ERK phosphorylation as well as
CF differentiation (Fig. 5). Overall, these results suggest the involvement of the MAPK/ERK pathway in TGF- $\beta$-induced CF differentiation into myofibroblasts. However, N-acetyl-L-cysteine (NAC), 


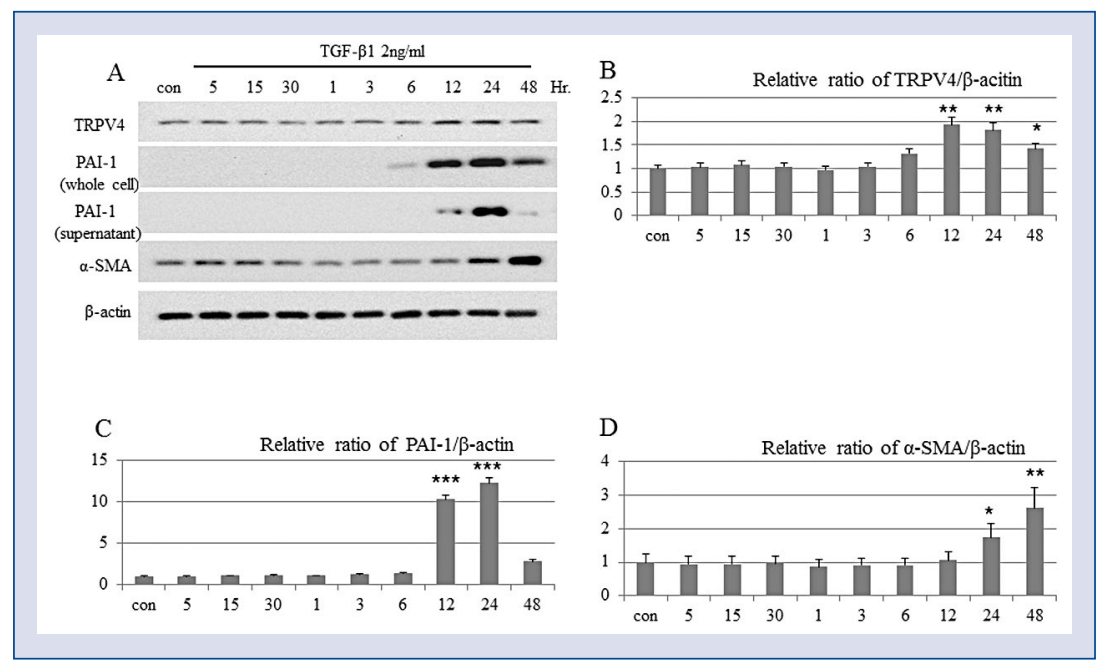

Figure 3. Transforming growth factor beta (TGF- $\beta$ ) induces TRPV4 protein expression and differentiation of ventricular fibroblasts. Cardiac fibroblasts were treated with $2 \mathrm{ng} / \mathrm{mL}$ TGF- $\beta 1$ and analyzed for protein expression of TRPV4, smooth muscle actin alpha ( $\alpha$-SMA), and plasminogen activator inhibitor-1 (PAI-1) by Western blotting; A. Representative Western blotting image; B-D. Relative protein expression of TRPV (B), plasminogen activator inhibitor-1 (PAI-1) (C), and smooth muscle actin alpha $\left(\alpha\right.$-SMA) (D) after TGF- $\beta$ treatment $\left({ }^{*} p<0.05\right.$, ${ }^{*} p<0.01$, and *** $p<0.001$, respectively, vs. untreated cells); con - control.

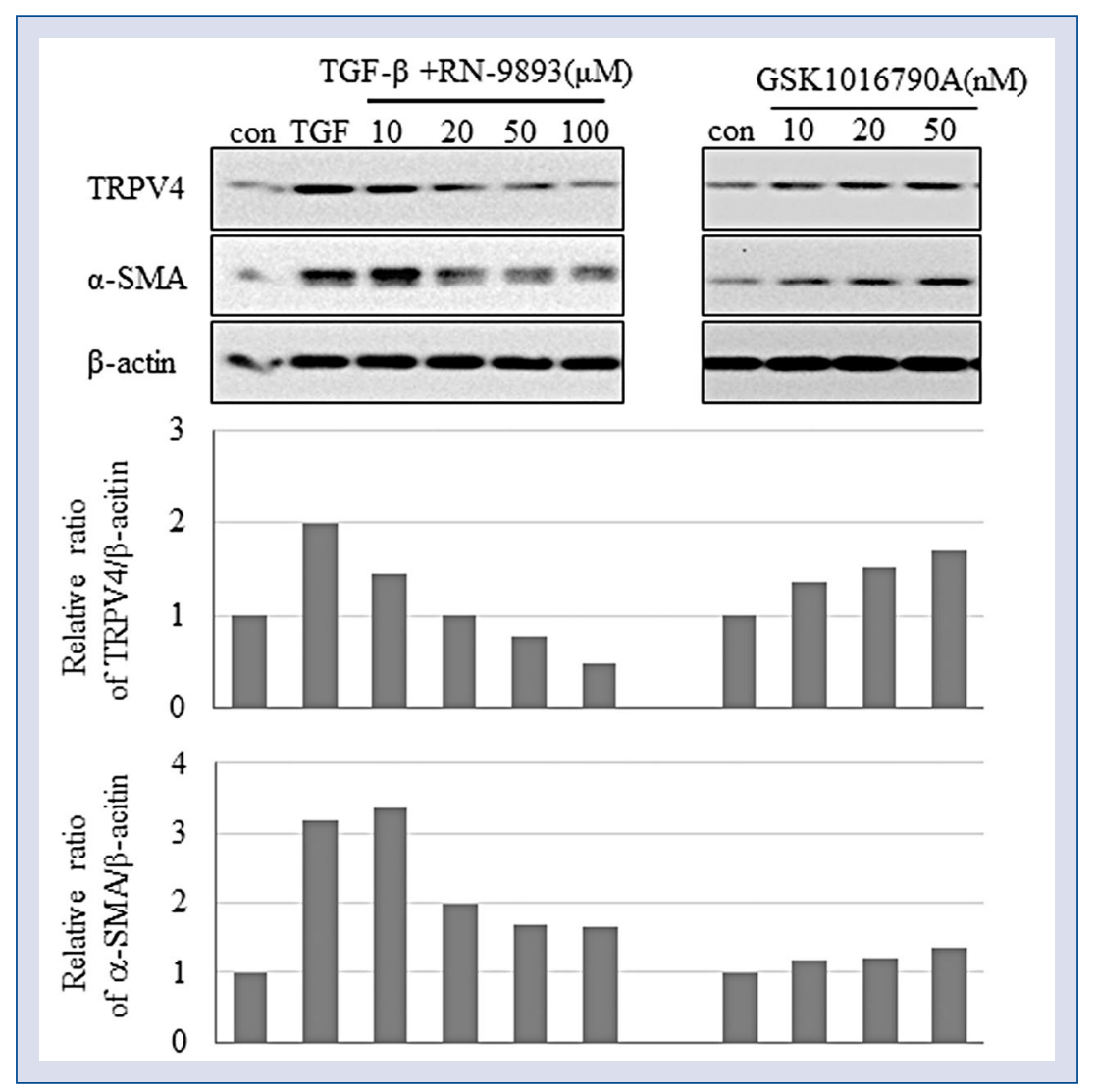

Figure 4. Transient receptor potential V4 (TRPV4) is involved in fibroblast differentiation. Cardiac fibroblasts (CFs) were pre-treated with the indicated concentrations of TRPV4 agonist GSK1016790A or antagonist RN-9893 and then treated with $2 \mathrm{ng} / \mathrm{mL}$ Transforming growth factor beta (TGF- $\beta$ ) for $24 \mathrm{~h}$. CF conversion into myofibroblasts was assessed based on smooth muscle actin alpha ( $\alpha$-SMA) expression analyzed by Western blotting. 


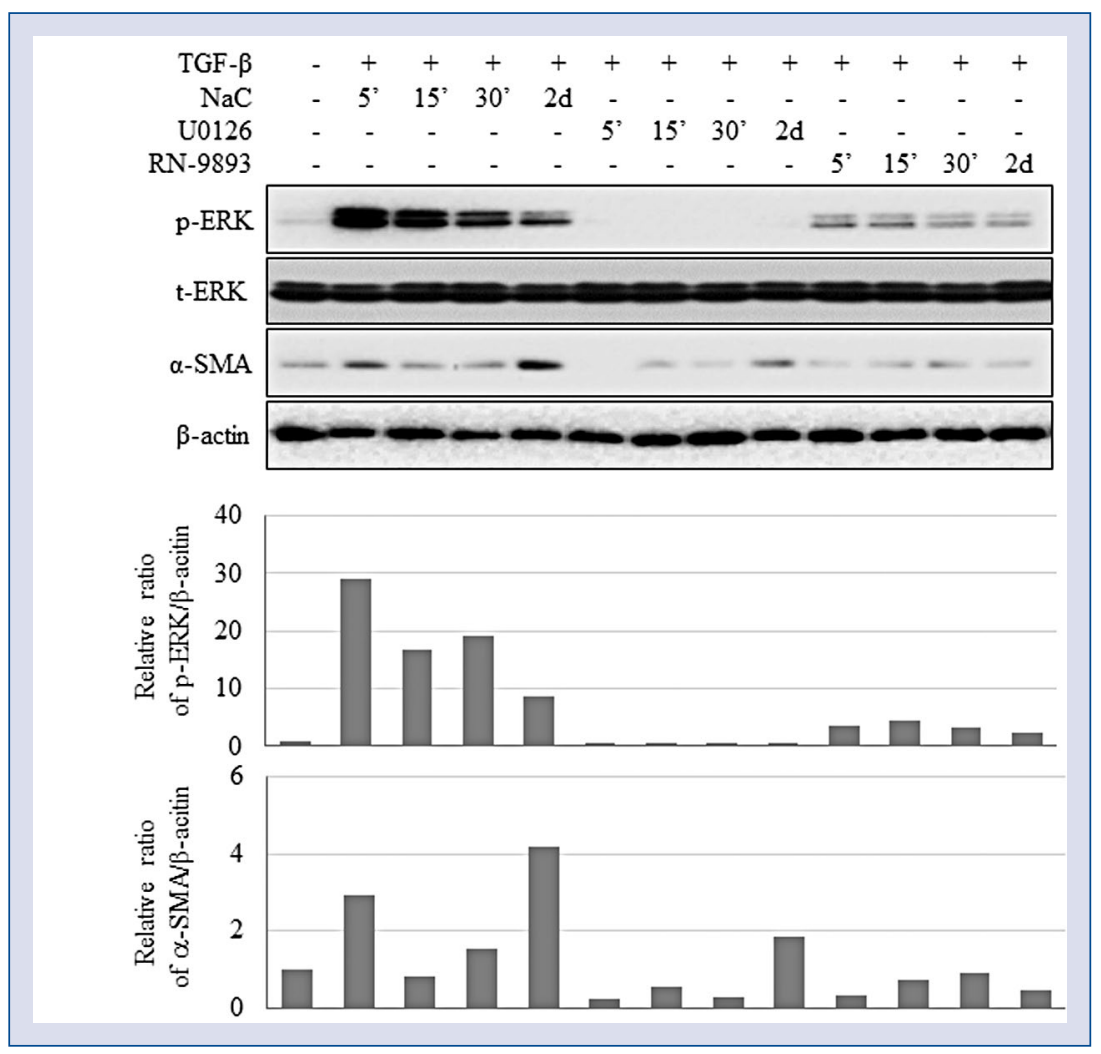

Figure 5. ERK activation is involved in TRPV4-mediated differentiation of human ventricular fibroblasts. Cardiac fibroblasts (CFs) were treated with $2 \mathrm{ng} / \mathrm{mL}$ of transforming growth factor beta (TGF- $\beta$ ) in the presence or absence of selective ERK inhibitor U0126 $(10 \mu \mathrm{M})$, reactive oxygen species scavenger N-acetyl-L-cysteine (NAC $0.5 \mathrm{mM})$, or TRPV4 antagonist RN-9893 (50 $\mu \mathrm{M})$ and analyzed for the expression of p-ERK (T202/Y204) and smooth muscle actin alpha ( $\alpha$-SMA).

a well-known scavenger of ROS did not influence either ERK activation or CF differentiation (Fig. 5).

\section{TRPV4 is essential for $\mathrm{Ca}^{2+}$ entry into} human ventricular fibroblasts

To analyze $\mathrm{Ca}^{2+}$ influx into differentiating $\mathrm{CFs}$, cytosolic $\mathrm{Ca}^{2+}$ levels were measured using a ratiometric fluorescent dye Fura2. Intracellular $\mathrm{Ca}^{2+}$ concentration increased in $\mathrm{CFs}$ after treatment with the TRPV4 agonist as well as with TGF- $\beta$. Given that both TRPV4 and TGF- $\beta$ induced CF differentiation, these results suggest that TRPV4 as a $\mathrm{Ca}^{2+}$-permeable channel has a role in the differentiation of human CFs into myofibroblasts (Fig. 6).

\section{Discussion}

In the current study, the pathophysiological function of the $\mathrm{Ca}^{2+}$-permeable channel TRPV4 in human ventricular CFs were under investigation. Results confirm that $\mathrm{Ca}^{2+}$ is essential for $\mathrm{CF}$ differentiation into myofibroblasts; most importantly, they also revealed that TGF- $\beta$ upregulates
TRPV4 expression and that TRPV4 mediates TGF- $\beta$-induced differentiation of human ventricular CFs into myofibroblasts via $\mathrm{Ca}^{2+}$-dependent ERK phosphorylation.

$\mathrm{Ca}^{2+}$ signaling is a ubiquitous intracellular mechanism responsible for diverse cellular functions [8]. Several studies suggest that $\mathrm{Ca}^{2+}$ is essential for $\mathrm{CF}$ proliferation and differentiation. The mitogenic effect of substance $P$ in rat $C F$ s was abolished by chelation of extracellular $\mathrm{Ca}^{2+}$ with EGTA, which inhibited cell proliferation [20]. Furthermore, a $\mathrm{Ca}^{2+}$ channel blocker prevented angiotensin II-mediated myocardial fibrosis and accumulation of myofibroblasts in vivo [21]. Consistent with these findings, the present results indicate that TGF- $\beta$ -induced human ventricular $\mathrm{CF}$ differentiation into myofibroblasts was inhibited by intracellular $\mathrm{Ca}^{2+}$ chelation. Overall, these data prove that $\mathrm{Ca}^{2+}$ entry through $\mathrm{Ca}^{2+}$-permeable ion channels are important for fibroblast gene expression and differentiation.

Fibroblasts are electrically non-excitable cells and have high membrane resistance and a relatively depolarized resting membrane potential of 


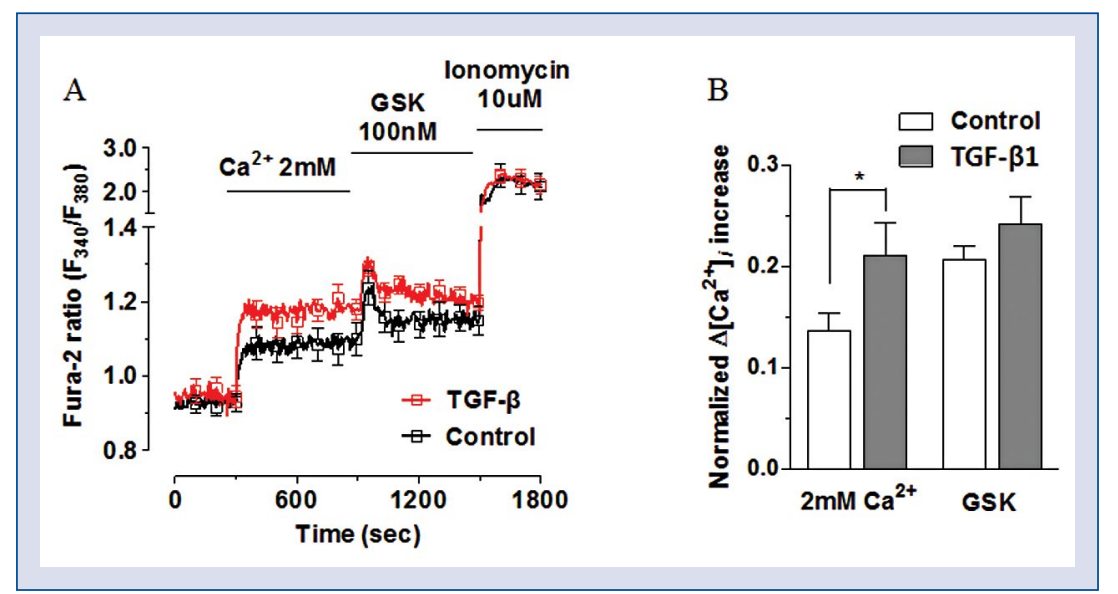

Figure 6. TRPV4 mediates $\mathrm{Ca}^{2+}$ influx required for cardiac fibroblasts (CFs) differentiation; A. Ca ${ }^{2+}$ influx was elicited by $2 \mathrm{mM} \mathrm{Ca}^{2+}$ and further augmented by the treatment with TRPV4 agonist GSK1016790A; B. Ca ${ }^{2+}$ influx was compared between control CFs (clear bar) and transforming growth factor beta (TGF- $\beta$ )-treated CFs (gray bar); the treatment with TRPV4 agonist GSK1016790A further increased $\mathrm{Ca}^{2+}$ influx ( ${ }^{*} \mathrm{p}<0.05$ vs. control).

$-15.9 \pm 2.1 \mathrm{mV}$ [22]. CFs typically do not express voltage-gated $\mathrm{Ca}^{2+}$ channels including those of L-type characteristic for cardiomyocytes [23] and are known to be mechanosensitive [10]. Therefore, $\mathrm{CF}$ s need a different $\mathrm{Ca}^{2+}$-signaling pathway to facilitate differentiation into myofibroblasts. The TRP superfamily of ion channels comprises nonselective cation channels permeable for $\mathrm{Ca}^{2+}$ and $\mathrm{Mg}^{2+}$. There are 28 mammalian TRP genes divided into six subfamilies based on amino acid homology: TRPC (Canonical), TRPV (Vanilloid), TRPM (Melastatin), TRPA (Ankyrin), TRPP (Polycystin), and TRPML (Mucolipin). Among these, all TRPC and TRPV channels as well as TRPM1, 2, 3, 6, 7, and 8 , TRPA1, TRPP2, 3 , and 5, and TRPML1, 2 , and 3 are permeable for $\mathrm{Ca}^{2+}$ [24]. Several previous studies indicate that TRP channels are involved in $\mathrm{Ca}^{2+}$-dependent regulation of $\mathrm{CF}$ differentiation into myofibroblasts in different mammalian species. Thus, Du et al. [14] suggested that TRPM7 played a major role in $\mathrm{Ca}^{2+}$ entry into human atrial fibroblasts by showing that the expression of TRPM7 mRNA was increased by TGF- $\beta$ which required a TRPM7-mediated $\mathrm{Ca}^{2+}$ signal for its effect on $\mathrm{CF}$ proliferation and differentiation. Other investigators showed that the proliferation and differentiation of rat CFs is regulated by $\mathrm{Ca}^{2+}$ influx through TRPC3 [15], whereas TRPC6 was necessary for the transformation of mouse embryonic CFs into myofibroblasts [13]. Furthermore, it has been demonstrated that a mechanosensitve channel TRPV4 is required for TGF- $\beta$-induced differentiation of adult rat CFs into myofibroblasts and that TGF- $\beta$ upregulated TRPV4 expression and TRPV4-mediated $\mathrm{Ca}^{2+}$ influx [12]. These findings indicate that TRP channels regulate CF differentiation into myofibroblasts, but their activity is species-and tissue-specific. The present study is the first to investigate the role of TRP channels in human ventricular CFs and the obtained results indicate that TRPV4 is the channel involved in TGF- $\beta$-induced conversion of human ventricular CFs into myofibroblasts. TRPV4 expression was upregulated by TGF- $\beta$ both at the mRNA and protein levels, which correlated with the induction of $\mathrm{Ca}^{2+}$ influx as well as myofibroblast transdifferentiation. At the same time, TRPV4 inhibition suppressed, while its activation induced the differentiation of CFs, confirming the regulatory role of TRPV4 in the process.

Transient receptor potential channels belonging to different subfamilies induce distinct downstream signaling mechanisms. TRPC6 was shown to upregulate $\mathrm{Ca}^{2+}$-responsive protein phosphatase calcineurin through its downstream transcriptional effector, nuclear factor of activated T-cells (NFAT), which resulted in myofibroblast trans-differentiation, whereas TRPC 6 expression was induced by TGF- $\beta$ through the MAPK/p38 pathway [13]. It has been suggested that TRPC3 expression mediating $\mathrm{CF}$ differentiation could be via a potential downstream mechanism involving the activation of ERK1/2 [15]. Protein kinases ERK1/2 are key intracellular signaling molecules regulated by phosphorylation and implicated in numerous biological processes, including cell growth, 


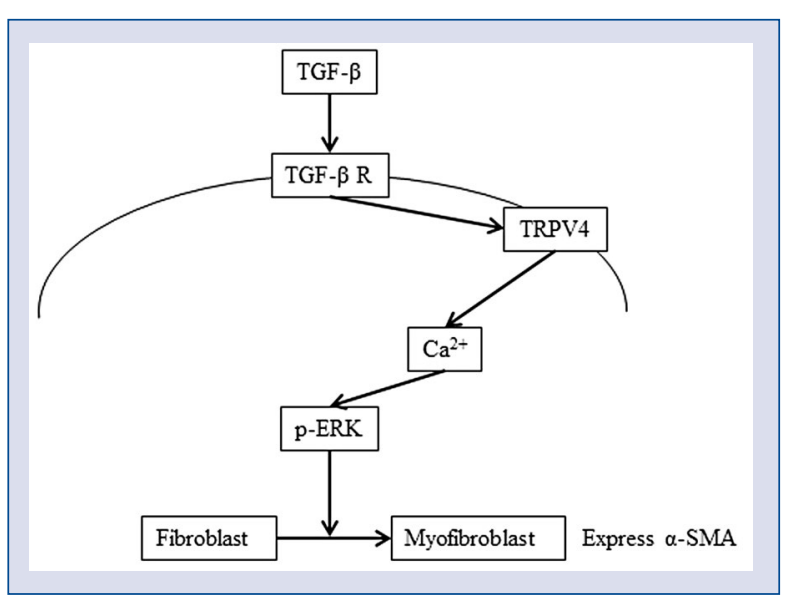

Figure 7. A signaling model for TRPV4-mediated differentiation of human ventricular fibroblasts. Transforming growth factor beta (TGF- $\beta$ ) signaling upregulates the expression of TRPV4, which induces $\mathrm{Ca}^{2+}$ influx and ERK $1 / 2$ activation, ultimately resulting in cardiac fibroblasts conversion into myofibroblasts; $\alpha$-SMA smooth muscle actin alpha.

survival, and differentiation. In the current study, $\mathrm{CF}$ differentiation and ERK1/2 phosphorylation were increased by TGF- $\beta$, but were decreased by the intracellular $\mathrm{Ca}^{2+}$ chelator, TRPV4 antagonist, and ERK inhibitor, indicating the involvement of ERK1/2 in TRPV4-dependent conversion of ventricular CFs into myofibroblasts.

Based on the current findings, the following mechanism underlying fibroblast trans-differentiation in the ventricle can be suggested: TGF- $\beta$ secreted by inflammatory cells after heart injury upregulates the expression of TRPV4 and $\mathrm{Ca}^{2+}$ influx, which activates the MAPK/ERK pathway and downstream transcription of the genes encoding myofibroblast markers such as $\alpha$-SMA, leading to a phenotypic switch from CFs to myofibroblasts (Fig. 7).

\section{Conclusions}

In conclusion, it was demonstrated that the $\mathrm{Ca}^{2+}$-permeable cation channel TRPV4 participated in $\mathrm{Ca}^{2+}$-mediated signaling in human ventricular fibroblasts, promoting their differentiation into myofibroblasts through the MAPK/ERK pathway. Findings herein, indicate that TRPV4 plays an important biological role in the regulation of left ventricular function and fibrogenesis in the heart, suggesting TRPV4 as a potential molecular target to attenuate cardiac fibrosis.

\section{Conflict of interest: None declared}

\section{References}

1. Jourdan-Lesaux C, Zhang J, Lindsey ML. Extracellular matrix roles during cardiac repair. Life Sci. 2010; 87(13-14): 391-400, doi: 10.1016/j.lfs.2010.07.010, indexed in Pubmed: 20670633.

2. Swynghedauw B. Molecular mechanisms of myocardial remodeling. Physiol Rev. 1999; 79(1): 215-262, doi: 10.1152/physrev.1999.79.1.215, indexed in Pubmed: 9922372.

3. Sun Y, Weber KT. Infarct scar: a dynamic tissue. Cardiovasc Res. 2000; 46(2): 250-256, indexed in Pubmed: 10773228.

4. Flack EC, Lindsey ML, Squires CE, et al. Alterations in cultured myocardial fibroblast function following the development of left ventricular failure. J Mol Cell Cardiol. 2006; 40(4): 474-483, doi: 10.1016/j.yjmcc.2006.01.019, indexed in Pubmed: 16516916.

5. Dobaczewski M, Chen W, Frangogiannis NG. Transforming growth factor (TGF) $-\beta$ signaling in cardiac remodeling. J Mol Cell Cardiol. 2011; 51(4): 600-606, doi: 10.1016/j.yjmcc.2010.10.033, indexed in Pubmed: 21059352.

6. Petrov VV, Fagard RH, Lijnen PJ. Stimulation of collagen production by transforming growth factor-beta1 during differentiation of cardiac fibroblasts to myofibroblasts. Hypertension. 2002; 39(2): 258-263, indexed in Pubmed: 11847194.

7. Tomasek JJ, Gabbiani G, Hinz B, et al. Myofibroblasts and mechano-regulation of connective tissue remodelling. Nat Rev Mol Cell Biol. 2002; 3(5): 349-363, doi: 10.1038/nrm809, indexed in Pubmed: 11988769

8. Berridge MJ, Bootman MD, Roderick HL. Calcium signalling: dynamics, homeostasis and remodelling. Nat Rev Mol Cell Biol. 2003; 4(7): 517-529, doi: 10.1038/nrm1155, indexed in Pubmed: 12838335.

9. Kamkin A, Kiseleva I, Wagner KD, et al. A possible role for atrial fibroblasts in postinfarction bradycardia. Am J Physiol Heart Circ Physiol. 2002; 282(3): H842-H849, doi: 10.1152/ajpheart.00240.2001, indexed in Pubmed: 11834477.

10. Kohl P, Noble D. Mechanosensitive connective tissue: potential influence on heart rhythm. Cardiovasc Res. 1996; 32(1): 62-68, indexed in Pubmed: 8776404.

11. Nilius B. TRP channels in disease. Biochim Biophys Acta. 2007; 1772(8): 805-812, doi: 10.1016/j.bbadis.2007.02.002, indexed in Pubmed: 17368864.

12. Adapala RK, Thoppil RJ, Luther DJ, et al. TRPV4 channels mediate cardiac fibroblast differentiation by integrating mechanical and soluble signals. J Mol Cell Cardiol. 2013; 54: 45-52, doi: 10.1016/j.yjmcc.2012.10.016, indexed in Pubmed: 23142541.

13. Davis J, Burr AR, Davis GF, et al. A TRPC6-dependent pathway for myofibroblast transdifferentiation and wound healing in vivo. Dev Cell. 2012; 23(4): 705-715, doi: 10.1016/j.devcel.2012.08.017, indexed in Pubmed: 23022034.

14. Du J, Xie J, Zhang $Z$, et al. TRPM7-mediated Ca2 + signals confer fibrogenesis in human atrial fibrillation. Circ Res. 2010; 106(5): 992-1003, doi: 10.1161/CIRCRESAHA.109.206771, indexed in Pubmed: 20075334.

15. Harada M, Luo X, Qi XY, et al. Transient receptor potential canonical-3 channel-dependent fibroblast regulation in atrial fibrillation. Circulation. 2012; 126(17): 2051-2064, doi: 10.1161/CIRCULATIONAHA.112.121830, indexed in Pubmed: 22992321.

16. Thodeti CK, Paruchuri S, Meszaros JG. A TRP to cardiac fibroblast differentiation. Channels (Austin). 2013; 7(3): 211-214, doi: 10.4161/chan.24328, indexed in Pubmed: 23511028.

17. Wei ZL, Nguyen MT, O'Mahony DJR, et al. Identification of orally-bioavailable antagonists of the TRPV4 ion-channel. Bioorg Med Chem Lett. 2015; 25(18): 4011-4015, doi: 10.1016/j. bmcl.2015.06.098, indexed in Pubmed: 26235950. 
18. Marshall ICB, Boyfield I, McNulty S. Ratiometric Ca2+ measurements using the FlexStation scanning fluorometer. Methods Mol Biol. 2006; 312: 119-124, indexed in Pubmed: 16422193.

19. Ghosh AK, Vaughan DE. PAI-1 in tissue fibrosis. J Cell Physiol. 2012; 227(2): 493-507, doi: 10.1002/jcp.22783, indexed in Pubmed: 21465481.

20. Kumaran C, Shivakumar K. Calcium- and superoxide anionmediated mitogenic action of substance $\mathrm{P}$ on cardiac fibroblasts. Am J Physiol Heart Circ Physiol. 2002; 282(5): H1855-H1862, doi: 10.1152/ajpheart.00747.2001, indexed in Pubmed: 11959652.

21. Ramires FJ, Sun Y, Weber KT. Myocardial fibrosis associated with aldosterone or angiotensin II administration: attenuation by calcium channel blockade. J Mol Cell Cardiol. 1998; 30(3): 475-483, doi: 10.1006/jmcc.1997.0612, indexed in Pubmed: 9515025.

22. Kamkin A, Kiseleva I, Wagner KD, et al. Mechanically induced potentials in fibroblasts from human right atrium. Exp Physiol. 1999; 84(2): 347-356, indexed in Pubmed: 10226175.

23. Bers DM. Cardiac excitation-contraction coupling. Nature. 2002; 415(6868): 198-205, doi: 10.1038/415198a, indexed in Pubmed: 11805843.

24. Pedersen SF, Owsianik G, Nilius B. TRP channels: an overview. Cell Calcium. 2005; 38(3-4): 233-252, doi: 10.1016/j. ceca.2005.06.028, indexed in Pubmed: 16098585. 